\title{
Eigenvalue correlations in non-Hermitean symplectic random matrices
}

\author{
Eugene Kanzieper 1 \\ Hitachi Cambridge Laboratory, Madingley Road, Cambridge CB3 0HE, United \\ Kingdom, and \\ Department of Condensed Matter Physics, Weizmann Institute of Science, \\ Rehovot 76100, Israel
}

\begin{abstract}
Correlation function of complex eigenvalues of $N \times N$ random matrices drawn from non-Hermitean random matrix ensemble of symplectic symmetry is given in terms of a quaternion determinant. Spectral properties of Gaussian ensembles are studied in detail in the regimes of weak and strong non-Hermiticity.
\end{abstract}

\section{Introduction}

Statistical ensembles of generic real, complex and quaternion matrices have first been introduced in the pioneering work by Ginibre (1965) who has managed to derive the joint probability distribution function (j.p.d.f.) of $N$ complex eigenvalues $\left\{z_{\ell}\right\}=\left\{x_{\ell}+i y_{\ell}\right\}$ of $N \times N$ complex $(\beta=2)$ and quaternion $(\beta=4)$ non-Hermitean random matrices:

$$
\begin{aligned}
& P_{N}^{(2)}\left(z_{1}, \cdots, z_{N}\right)=C_{2}(N) \prod_{k<\ell}\left|z_{k}-z_{\ell}\right|^{2} \prod_{\ell=1}^{N} w^{2}\left(z_{\ell}, \bar{z}_{\ell}\right), \\
& P_{N}^{(4)}\left(z_{1}, \cdots, z_{N}\right)=C_{4}(N) \prod_{k<\ell}\left|z_{k}-z_{\ell}\right|^{2}\left|z_{k}-\bar{z}_{\ell}\right|^{2} \prod_{\ell=1}^{N}\left|z_{\ell}-\bar{z}_{\ell}\right|^{2} w^{2}\left(z_{\ell}, \bar{z}_{\ell}\right) .
\end{aligned}
$$

$C_{\beta}(N)$ is a normalisation constant, $w^{2}(z, \bar{z})$ is a weight function (see discussion below). For real matrices $(\beta=1)$ with no further symmetries, the reader is referred to much later papers by Lehmann and Sommers (1991), and also by Edelman (1997).

Although Ginibre's derivation of Eqs. (1) and (2) holds for random matrices with Gaussian distributed entries, that is for

$$
w^{2}(z, \bar{z})=w_{0}^{2}(z, \bar{z})=e^{-z \bar{z}}
$$

we will allow the weight $w^{2}(z, \bar{z})$ to be an arbitrary benign function of $z$ and $\bar{z}$ provided the normalisation $C_{\beta}(N)$ exists. It should be emphasized ${ }_{3}$ that such an innocent (at first glance) extension is quite nontrivial as it raises the question about existence of an underlying matrix model whose eigenvalue representation would coincide with Eqs. (1) and (2). Say, if the underlying non-Hermitean matrix commutes with its adjoint (such matrices are often called normal matrices, see, e.g., Oas, 1997), the interpretation of

$\dagger$ E-mail address: eugene@phy.cam.ac.uk / eugene.kanzieper@weizmann.ac.il

$\S$ The referee is thanked for pointing this out. 
Eqs. (11) and (2) as a non-Gaussian j.p.d.f is correct. Notice, however, that such a commutativity constraint is not a must though. For example, an ensemble of weakly non-Hermitean matrices introduced by Fyodorov, Khoruzhenko and Sommers (1997) is described by j.p.d.f. of the form (11) and (2), see Fyodorov, Khoruzhenko and Sommers (1998) and Hastings (2000).

Of particular interest is the $n$-point correlation function which describes a probability density to find $n$ complex eigenvalues around each of the points $z_{1}, \cdots, z_{n}$ while positions of the remaining levels are unobserved:

$$
R_{n}^{(\beta)}\left(z_{1}, \ldots, z_{n}\right)=\frac{N !}{(N-n) !} \int \cdots \int d^{2} Z_{n+1} \cdots d^{2} Z_{N} P_{N}^{(\beta)}\left(z_{1}, \cdots, z_{N}\right) .
$$

The integration measure $d^{2} Z_{\ell}$ is $d^{2} Z_{\ell}=d x_{\ell} d y_{\ell}$. Quite often, one is also interested in a thermodynamic limit

$$
\rho_{n}^{(\beta)}\left(z_{1}, \cdots, z_{n}\right)=\lim _{N \rightarrow \infty} \frac{1}{\delta_{N}^{2 n}} R_{n}^{(\beta)}\left(\frac{z_{1}}{\delta_{N}}, \cdots, \frac{z_{n}}{\delta_{N}}\right)
$$

which magnifies spectrum resolution on the appropriate energy scale $\delta_{N}$ while letting matrix size $N$ tend to infinity.

At $\beta=2$, the $n$-point correlation function for non-Hermitean matrix model has also been studied by Ginibre (1965). Adopting the method of orthogonal polynomials introduced in the context of Hermitean random matrix theory by Mehta and Gaudin (1960), it is a straightforward exercise to demonstrate that $R_{n}^{(2)}\left(z_{1}, \cdots, z_{n}\right)$ admits the determinant representation

$$
R_{n}^{(2)}\left(z_{1}, \cdots, z_{n}\right)=\operatorname{det}\left[K_{N}^{(2)}\left(z_{k}, \bar{z}_{\ell}\right)\right]_{k, \ell=1, \cdots, n} .
$$

The scalar kernel

$$
K_{N}^{(2)}\left(z, z^{\prime}\right)=w(z, \bar{z}) w\left(z^{\prime}, \bar{z}^{\prime}\right) \sum_{k=0}^{N-1} P_{k}(z) P_{k}\left(z^{\prime}\right)
$$

is expressed in terms of polynomials $P_{k}(z)$ orthonormal in the complex plane $z=x+i y$

$$
\int d^{2} Z w^{2}(z, \bar{z}) P_{k}(z) P_{\ell}(\bar{z})=\delta_{k \ell}
$$

with respect to the measure $w^{2}(z, \bar{z}) d^{2} Z$.

For instance, the density of states and the two-point correlation function equal

$$
R_{1}^{(2)}(z)=K_{N}(z, \bar{z})
$$

and

$$
R_{2}^{(2)}\left(z_{1}, z_{2}\right)=K_{N}\left(z_{1}, \bar{z}_{1}\right) K_{N}\left(z_{2}, \bar{z}_{2}\right)-\left|K_{N}\left(z_{1}, \bar{z}_{2}\right)\right|^{2},
$$

respectively.

Non-Hermitean random matrices at $\beta=4$ have also received some attention in both physical and mathematical literature especially following a recent burst of interest to spectral properties of non-Hermitean random operators (see, e.g., Efetov (1997)).

M. L. Mehta (1967) considered a non-Hermitean matrix model of symplectic symmetry for Ginibre's weight function $w_{0}^{2}(z, \bar{z})=\exp (-z \bar{z})$, and established a quaternion determinant structure of one- and two-point correlation functions. He also conjectured a similar structure to hold for all $n$-point correlation functions. In Ginibre's case, these appear in the revised 1991 edition of the Mehta's book. 
Further progress has come with development of field theoretic techniques. Kolesnikov and Efetov (1997), driven by possible applications in quantum chromodynamics (Halasz, Osborn and Verbaarschot, 1997), have formulated a nonlinear supersymmetry $\sigma$-model for this class of random matrices, and derived an expression for the eigenvalue density in a somewhat richer model [see Eq. (21) below].

More recently, yet another field theory approach (aka replica method) was outlined by Nishigaki and Kamenev (2002). There, the well known Mehta's expressions were reproduced for one-point correlation function in case of the very same Ginibre's weight function $w_{0}^{2}(z, \bar{z})$. Unfortunately, both mentioned techniques run into obstacles when one attempts to study higher-order correlation functions whilst replica $\sigma$-models seem to reliably provide asymptotic expansions only.

A different route has been chosen by Hastings (2000) who suggested that there exists a mapping of non-Hermitean random matrices of symplectic symmetry onto a fermion field theory. Even though the method might have been potentially applicable to a study of $n$-point correlation functions in the bulk of a complex spectrum, these have not explicitly been worked out beyond the two-point correlation function.

Our paper reports on a comprehensive treatment of integrable structure of nonHermitean random matrix models at $\beta=4$. It sets a transparent and coherent framework to study all $n$-point correlation functions: while easily applied to reproduce the results of aforementioned studies and extend them to higher-order correlation functions in the spectrum bulk, it may go much farther and serve as a proper starting point to explore eigenvalue correlations near the spectrum edges and/or address the issue of universality (for a recent review of the universality phenomenon in the context of Hermitean random matrix models see, e.g., Kanzieper and Freilikher, 1999).

The paper is organised as follows. Section 2 announces a most general form of $n$-point correlation function whatever the weight function in Eq. (2) is. A proof is given in Section 3. A concept of skew orthogonal polynomials which are central to performing explicit calculations is elaborated in detail in Section 4. There, exact expressions for skew orthogonal polynomials are given in terms of multi-fold integrals. For the Gaussian weight Eq. (21), the polynomials are evaluated explicitly. In Section $5, n$-point correlation functions for the Gaussian weight [Eq. (21)] are derived for finite $N$ as well as in the large- $N$ limit. Section 6 contains concluding remarks and briefly mentions further possible applications of the formalism developed.

\section{Correlation function at $\beta=4$ and eigenvalue depletion along real axis}

For symplectic ensemble, the following representation holds for $n$-point correlation function:

$$
R_{n}^{(4)}\left(z_{1}, \ldots, z_{n}\right)=Q \operatorname{det}\left[K_{N}^{(4)}\left(z_{k}, z_{\ell}\right)\right]_{k, \ell=1, \cdots, n} .
$$

Here $Q$ det stands for a quaternion determinant (Dyson, 1972). The $2 \times 2$ matrix kernel

$$
\begin{aligned}
K_{N}^{(4)}\left(z, z^{\prime}\right) & =(\bar{z}-z)^{1 / 2}\left(\bar{z}^{\prime}-z^{\prime}\right)^{1 / 2} w(z, \bar{z}) w\left(z^{\prime}, \bar{z}^{\prime}\right) \\
& \times\left(\begin{array}{cc}
\kappa_{N}\left(\bar{z}, z^{\prime}\right) & -\kappa_{N}\left(\bar{z}, \bar{z}^{\prime}\right) \\
\kappa_{N}\left(z, z^{\prime}\right) & -\kappa_{N}\left(z, \bar{z}^{\prime}\right)
\end{array}\right)
\end{aligned}
$$

where the 'prekernel' $\kappa_{N}$ is

$$
\kappa_{N}\left(z, z^{\prime}\right)=\sum_{k, \ell=0}^{2 N-1} p_{k}(z)\left(M^{-1}\right)_{k \ell} p_{\ell}\left(z^{\prime}\right),
$$


and $M^{-1}$ is an inverse to the real antisymmetric matrix $M$ with the entries

$$
M_{k \ell}=\int d^{2} Z(\bar{z}-z) w^{2}(z, \bar{z})\left[p_{k}(z) p_{\ell}(\bar{z})-p_{\ell}(z) p_{k}(\bar{z})\right] .
$$

The polynomials $p_{k}(z)$ are arbitrary provided the inverse $M^{-1}$ exists. Since the matrix $M$ is antisymmetric, the formulas would be simplest had it contained $N$ copies of the $2 \times 2$ matrix

$$
i \sigma_{y}=\left(\begin{array}{cc}
0 & 1 \\
-1 & 0
\end{array}\right)
$$

along the main diagonal. This is achieved by letting $p_{k}(z)$ be skew-orthogonal polynomials $q_{k}(z)$ in the complex domain:

$$
\begin{aligned}
& \left\langle q_{2 k+1}, q_{2 \ell}\right\rangle_{S}=-\left\langle q_{2 \ell}, q_{2 k+1}\right\rangle_{S}=r_{k} \delta_{k \ell}, \\
& \left\langle q_{2 k+1}, q_{2 \ell+1}\right\rangle_{S}=\left\langle q_{2 k}, q_{2 \ell}\right\rangle_{S}=0 .
\end{aligned}
$$

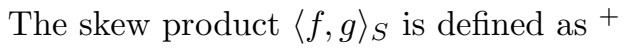

$$
\langle f, g\rangle_{S}=\int d^{2} Z(\bar{z}-z) w^{2}(z, \bar{z})[f(z) g(\bar{z})-f(\bar{z}) g(z)] .
$$

With this choice in mind, the prekernel $\kappa_{N}$ further simplifies to

$$
\kappa_{N}\left(z, z^{\prime}\right)=\sum_{k=0}^{N-1} \frac{q_{2 k+1}(z) q_{2 k}\left(z^{\prime}\right)-q_{2 k+1}\left(z^{\prime}\right) q_{2 k}(z)}{r_{k}} .
$$

In particular, the density of states and the two-point correlation functions are expressed as

$$
R_{1}^{(4)}(z)=(\bar{z}-z) w^{2}(z, \bar{z}) \kappa_{N}(z, \bar{z})
$$

and

$$
\begin{aligned}
R_{2}^{(4)}\left(z_{1}, z_{2}\right) & =\left(\bar{z}_{1}-z_{1}\right)\left(\bar{z}_{2}-z_{2}\right) w^{2}\left(z_{1}, \bar{z}_{1}\right) w^{2}\left(z_{2}, \bar{z}_{2}\right) \\
& \times\left[\kappa_{N}\left(z_{1}, \bar{z}_{1}\right) \kappa_{N}\left(z_{2}, \bar{z}_{2}\right)-\left|\kappa_{N}\left(z_{1}, z_{2}\right)\right|^{2}+\left|\kappa_{N}\left(z_{1}, \bar{z}_{2}\right)\right|^{2}\right],
\end{aligned}
$$

respectively.

Notice that, in accordance with our solution [Eqs. (6) and (7)], the $n$-point correlation function universally vanishes along the real axes $\Im \mathrm{m} z_{\ell}=0$,

$$
R_{n}^{(4)}\left(z_{1}, \cdots, z_{n}\right) \propto \prod_{\ell=1}^{n}\left[\Im \mathrm{m} z_{\ell}\right]^{\alpha}, \quad \alpha \equiv 2
$$

whatever the weight function $w^{2}(z, \bar{z})$ is. It is this specific feature of spectral correlations in symplectic ensembles of non-Hermitean random matrices that has been revealed, for $n=1$, in early numerical simulations due to Halasz, Osborn and Verbaarschot (1997). Qualitatively, such a depletion of complex eigenvalues along the real axis might have been anticipated after a brief inspection of both the j.p.d.f. [Eq. (2)] and the definition of $n$-point correlation function [Eq. (4)].

The results announced [Eqs. (6) - (13)] will be derived in Section 3. In Section 4 , we study properties of skew-orthogonal polynomials which constitute a natural basis to perform actual calculations of spectral fluctuations in $\beta=4$ non-Hermitean random matrix ensembles. The latter are addressed in Section 5 , where we consider an

+ Notice a difference from the skew orthogonality arising in the context of Hermitean random matrices (Mahoux and Mehta, 1991). 
ensemble of $N \times N$ random matrices associated with a Gaussian measure. Correlation functions in the regimes of strong (Ginibre, 1965) and weak (Fyodorov, Khoruzhenko and Sommers, 1997) non-Hermiticity are explicitly given there for finite $N$ as well as in the limit of infinite matrices.

\section{Derivation}

To derive a quaternion determinant representation of $n$-point correlation function, we will follow an elegant idea of Tracy and Widom (1998). These authors have introduced generating functional

$$
G[f]=\int \cdots \int d^{2} Z_{1} \cdots d^{2} Z_{N} P_{N}\left(z_{1}, \cdots, z_{N}\right) \prod_{k=1}^{N}\left[1+f\left(z_{k}\right)\right]
$$

such that the $n$-point correlation function $R_{n}\left(z_{1}, \cdots, z_{n}\right)$ defined by Eq. (舟) can be viewed as the coefficient of $\alpha_{1} \cdots \alpha_{n}$ in the expansion of $G[f]$ for a particular choice $f(z)=\sum_{r=1}^{N} \alpha_{r} \delta^{2}\left(z-z_{r}\right)$. Assuming that $G[f]$ admits the representation

$$
G[f]=\sqrt{\operatorname{det}\left(I+K_{N} f\right)},
$$

where $K_{N}$ denotes the operator with $2 \times 2$ (self-dual) matrix kernel $K_{N}\left(z, z^{\prime}\right)$ and $f$ denotes multiplication by that function, Tracy and Widom have explicitly evaluated the coefficient of $\alpha_{1} \cdots \alpha_{n}$ and found it to be equal to the quaternion determinant in the r.h.s. of Eq. (5).

Hence, in accordance with this statement (which we will name the Tracy-Widom theorem) one has to seek a suitable representation for $G[f]$ with the j.p.d.f. given by Eq. (2). This is easy. Due to the identity

$$
\prod_{k<\ell}\left(x_{k}-x_{\ell}\right) \prod_{k, \ell=1}^{N}\left(y_{k}-x_{\ell}\right) \prod_{k>\ell}\left(y_{k}-y_{\ell}\right)=\operatorname{det}\left[\begin{array}{c}
x_{\ell}^{k-1} \\
y_{\ell}^{k-1}
\end{array}\right]_{\substack{k=1, \cdots, 2 N \\
\ell=1, \cdots, N}}
$$

one notices that

$$
\prod_{k<\ell}\left|z_{k}-z_{\ell}\right|^{2}\left|z_{k}-\bar{z}_{\ell}\right|^{2} \prod_{\ell=1}^{N}\left(\bar{z}_{\ell}-z_{\ell}\right)=\operatorname{det}\left[\begin{array}{c}
z_{\ell}^{k-1} \\
\bar{z}_{\ell}^{k-1}
\end{array}\right]_{\substack{k=1, \cdots, 2 N \\
\ell=1, \cdots, N}} .
$$

To derive the latter, we have put $x_{\ell}=z_{\ell}$ and $y_{\ell}=\bar{z}_{\ell}$ in the former. With this result in mind, the j.p.d.f. $P_{N}^{(4)}$ can be cast into the form

$$
\begin{aligned}
P_{N}^{(4)}\left(z_{1}, \cdots, z_{N}\right) & =C_{4}(N) \prod_{\ell=1}^{N}\left(z_{\ell}-\bar{z}_{\ell}\right) w^{2}\left(z_{\ell}, \bar{z}_{\ell}\right) \\
& \times \operatorname{det}\left[\begin{array}{l}
p_{k-1}\left(z_{\ell}\right) \\
p_{k-1}\left(\bar{z}_{\ell}\right)
\end{array}\right]_{\substack{k=1, \cdots, 2 N \\
\ell=1, \cdots, N}} .
\end{aligned}
$$

Here, we have replaced the sequence of monomials $\left\{z_{\ell}^{k}\right\}$ by arbitrary monic polynomials $\left\{p_{k}\left(z_{\ell}\right)\right\}$ of degree $k$ as this leaves the value of determinant intact. If the polynomials $p_{k}$ were not monic, the normalisation prefactor $C_{4}(N)$ would change. 
Eigenvalue correlations in non-Hermitean symplectic random matrices

This representation is fairly useful due to de Bruijn's (1955) integration formula

$$
\begin{aligned}
& \int \cdots \int d \alpha\left(Z_{1}\right) \cdots d \alpha\left(Z_{N}\right) \operatorname{det}\left[\begin{array}{l}
f_{k}\left(z_{\ell}\right) \\
g_{k}\left(z_{\ell}\right)
\end{array}\right]_{\substack{k=1, \cdots, 2 N \\
\ell=1, \cdots, N}} \\
& =(2 N) ! \operatorname{Pf}\left[\int d \alpha(Z)\left[f_{k}(z) g_{\ell}(z)-f_{\ell}(z) g_{k}(z)\right]\right]_{k, \ell=1, \cdots, 2 N}
\end{aligned}
$$

in which $d \alpha(Z)$ is an integration measure and 'Pf' stands for pfaffian. One derives the following chain:

$$
\begin{aligned}
G^{2}[f] & \propto \operatorname{det}\left[\int d^{2} Z(\bar{z}-z) w^{2}(z, \bar{z})\right. \\
& \left.\times[1+f(z)]\left[p_{k}(z) p_{\ell}(\bar{z})-p_{\ell}(z) p_{k}(\bar{z})\right]\right] \\
& \propto \operatorname{det}\left[M_{k \ell}+\int d^{2} Z(\bar{z}-z) w^{2}(z, \bar{z})\right. \\
& \left.\times f(z)\left[p_{k}(z) p_{\ell}(\bar{z})-p_{\ell}(z) p_{k}(\bar{z})\right]\right] \\
& \propto \operatorname{det}\left[\delta_{k \ell}+\int d^{2} Z(\bar{z}-z) w^{2}(z, \bar{z})\right. \\
& \left.\times f(z)\left[\pi_{k}(z) p_{\ell}(\bar{z})-p_{\ell}(z) \pi_{k}(\bar{z})\right]\right] .
\end{aligned}
$$

Matrix $M$ arisen in Eq. (16) is defined by Eq. (9). To derive Eq. (17), we have factored out $M$ on the left. Notice that Eq. (17) automatically bears a proper normalisation $G^{2}[0] \equiv 1$. The polynomials $\pi_{k}$ are

$$
\pi_{k}(z)=\sum_{\ell=0}^{2 N-1}\left(M^{-1}\right)_{k \ell} p_{\ell}(z) .
$$

The matrix appearing under the sign of determinant in Eq. 177 can be represented as $I+A B$ with

$$
\begin{aligned}
& A(\ell, z)=f(z)(\bar{z}-z)^{1 / 2} w(z, \bar{z})\left(\pi_{\ell}(z),-\pi_{\ell}(\bar{z})\right), \\
& B(z, \ell)=(\bar{z}-z)^{1 / 2} w(z, \bar{z})\left(\begin{array}{c}
p_{\ell}(\bar{z}) \\
p_{\ell}(z)
\end{array}\right) .
\end{aligned}
$$

As soon as the transposition does not affect the value of the determinant, one observes the identity $\operatorname{det}(I+A B)=\operatorname{det}(I+B A)$. In this case $B A$ is the integral operator with matrix kernel $K_{N}\left(z_{1}, z_{2}\right) f\left(z_{2}\right)$ where

$$
\begin{aligned}
K_{N}\left(z_{1}, z_{2}\right) & =\left(\bar{z}_{1}-z_{1}\right)^{1 / 2}\left(\bar{z}_{2}-z_{2}\right)^{1 / 2} w\left(z_{1}, \bar{z}_{1}\right) w\left(z_{2}, \bar{z}_{2}\right) \\
& \times\left(\begin{array}{ll}
\sum_{\ell} p_{\ell}\left(\bar{z}_{1}\right) \pi_{\ell}\left(z_{2}\right) & -\sum_{\ell} p_{\ell}\left(\bar{z}_{1}\right) \pi_{\ell}\left(\bar{z}_{2}\right) \\
\sum_{\ell} p_{\ell}\left(z_{1}\right) \pi_{\ell}\left(z_{2}\right) & -\sum_{\ell} p_{\ell}\left(z_{1}\right) \pi_{\ell}\left(\bar{z}_{2}\right)
\end{array}\right) .
\end{aligned}
$$

Hence, we have proven that $G^{2}[f]=\operatorname{det}\left(I+K_{N} f\right)$. Since the $2 \times 2$ matrix $K_{N}$ is self-dual| Eq. (6) follows by virtue of Tracy-Widom theorem. This completes our proof.

$\|$ Indeed, since the quaternion $\kappa_{N}$ is represented by $2 \times 2$ matrix

$$
\theta\left[\kappa_{N}\right]=\left(\begin{array}{ll}
\kappa_{N}\left(\bar{z}_{1}, z_{2}\right) & -\kappa_{N}\left(\bar{z}_{1}, \bar{z}_{2}\right) \\
\kappa_{N}\left(z_{1}, z_{2}\right) & -\kappa_{N}\left(z_{1}, \bar{z}_{2}\right)
\end{array}\right)
$$




\section{Skew-orthogonal polynomials}

\subsection{General weight $w^{2}(z, \bar{z})$}

We have seen in Section 2 that skew-orthogonal polynomials defined by Eqs. (10) - (12) represent a natural basis in which calculations become simplest. These can explicitly be found for a general weight function $w^{2}(z, \bar{z})$ provided the integrals below make sense and, in a monic normalisation, are given by the following $2 n$-fold integrals:

$$
\begin{aligned}
q_{2 n}(z) & \equiv \frac{1}{A_{n}} \int \cdots \int d^{2} Z_{1} \cdots d^{2} Z_{n} \prod_{\ell=1}^{n}\left(z-z_{\ell}\right)\left(z-\bar{z}_{\ell}\right) \\
& \times \prod_{k<\ell}\left|z_{k}-z_{\ell}\right|^{2}\left|z_{k}-\bar{z}_{\ell}\right|^{2} \prod_{\ell=1}^{n}\left|z_{\ell}-\bar{z}_{\ell}\right|^{2} w^{2}\left(z_{\ell}, \bar{z}_{\ell}\right), \\
q_{2 n+1}(z) & \equiv \frac{1}{A_{n}} \int \cdots \int d^{2} Z_{1} \cdots d^{2} Z_{n} \prod_{\ell=1}^{n}\left(z-z_{\ell}\right)\left(z-\bar{z}_{\ell}\right) \\
& \times\left(z+\sum_{k=1}^{n}\left(z_{k}+\bar{z}_{k}\right)+c_{n}\right) \\
& \times \prod_{k<\ell}\left|z_{k}-z_{\ell}\right|^{2}\left|z_{k}-\bar{z}_{\ell}\right|^{2} \prod_{\ell=1}^{n}\left|z_{\ell}-\bar{z}_{\ell}\right|^{2} w^{2}\left(z_{\ell}, \bar{z}_{\ell}\right) .
\end{aligned}
$$

Here $c_{n}$ is an arbitrary constant which we will set to zero, $c_{n}=0$ whilst

$$
A_{n}=\int \cdots \int d^{2} Z_{1} \cdots d^{2} Z_{n} \prod_{k<\ell}\left|z_{k}-z_{\ell}\right|^{2}\left|z_{k}-\bar{z}_{\ell}\right|^{2} \prod_{\ell=1}^{n}\left|z_{\ell}-\bar{z}_{\ell}\right|^{2} w^{2}\left(z_{\ell}, \bar{z}_{\ell}\right) .
$$

For similar representations of skew-orthogonal polynomials arisen in the context of Hermitean random matrix theory see Eynard (2001).

To prove that Eqs. (18) and (19) obey skew-orthogonality relations [Eqs. (10) - [12]], it is sufficient to show that (i) $\left\langle q_{2 n}, z^{m}\right\rangle_{S}=0$ and (ii) $\left\langle q_{2 n+1}, z^{m}\right\rangle_{S}=0$ for integer $0 \leq m \leq 2 n-1$.

(i) Consider

$$
\begin{aligned}
\left\langle q_{2 n}, z^{m}\right\rangle_{S} & \propto \int d^{2} Z(\bar{z}-z) w^{2}(z, \bar{z}) \int \cdots \int d^{2} Z_{1} \cdots d^{2} Z_{n} \\
& \times \prod_{k<\ell}\left|z_{k}-z_{\ell}\right|^{2}\left|z_{k}-\bar{z}_{\ell}\right|^{2} \prod_{\ell=1}^{n}\left|z_{\ell}-\bar{z}_{\ell}\right|^{2} w^{2}\left(z_{\ell}, \bar{z}_{\ell}\right) \\
& \times\left[\bar{z}^{m} \prod_{\ell=1}^{n}\left(z-z_{\ell}\right)\left(z-\bar{z}_{\ell}\right)-z^{m} \prod_{\ell=1}^{n}\left(\bar{z}-z_{\ell}\right)\left(\bar{z}-\bar{z}_{\ell}\right)\right] .
\end{aligned}
$$

As soon as

$$
\prod_{k<\ell}\left|z_{k}-z_{\ell}\right|^{2}\left|z_{k}-\bar{z}_{\ell}\right|^{2} \prod_{\ell=1}^{n}\left(\bar{z}_{\ell}-z_{\ell}\right) \prod_{\ell=1}^{n}\left(z-z_{\ell}\right)\left(z-\bar{z}_{\ell}\right)
$$

the dual quaternion $\tilde{\kappa}_{N}$ is given by

$$
\theta\left[\tilde{\kappa}_{N}\right]=\left(\begin{array}{ll}
-\kappa_{N}\left(z_{1}, \bar{z}_{2}\right) & \kappa_{N}\left(\bar{z}_{1}, \bar{z}_{2}\right) \\
-\kappa_{N}\left(z_{1}, z_{2}\right) & \kappa_{N}\left(\bar{z}_{1}, z_{2}\right)
\end{array}\right)
$$

Self-duality is a consequence of the equality $\sigma_{y} \theta\left[\tilde{\kappa}_{N}^{T}\right]=\theta\left[\kappa_{N}\right] \sigma_{y}$. 
Eigenvalue correlations in non-Hermitean symplectic random matrices

$$
=\operatorname{det}\left(\begin{array}{ccccc}
1 & z_{1} & \cdots & z_{1}^{2 n-1} & z_{1}^{2 n} \\
1 & \bar{z}_{1} & \cdots & \bar{z}_{1}^{2 n-1} & \bar{z}_{1}^{2 n} \\
\vdots & \vdots & & \vdots & \vdots \\
1 & z_{n} & \cdots & z_{n}^{2 n-1} & z_{n}^{2 n} \\
1 & \bar{z}_{n} & \cdots & \bar{z}_{n}^{2 n-1} & \bar{z}_{n}^{2 n} \\
1 & z & \cdots & z^{2 n-1} & z^{2 n}
\end{array}\right),
$$

we derive

$$
\begin{aligned}
\left\langle q_{2 n}, z^{m}\right\rangle_{S} & \propto \int \cdots \int d^{2} Z_{1} \cdots d^{2} Z_{n} d^{2} Z_{n+1} \prod_{\ell=1}^{n+1}\left(z_{\ell}-\bar{z}_{\ell}\right) w^{2}\left(z_{\ell}, \bar{z}_{\ell}\right) \\
& \times \operatorname{det}\left(\begin{array}{ccccc}
1 & z_{1} & \cdots & z_{1}^{2 n} & 0 \\
1 & \bar{z}_{1} & \cdots & \bar{z}_{1}^{2 n} & 0 \\
\vdots & \vdots & & \vdots & \vdots \\
1 & z_{n} & \cdots & z_{n}^{2 n} & 0 \\
1 & \bar{z}_{n} & \cdots & \bar{z}_{n}^{2 n} & 0 \\
1 & z_{n+1} & \cdots & z_{n+1}^{2 n} & z_{n+1}^{m} \\
1 & \bar{z}_{n+1} & \cdots & \bar{z}_{n+1}^{2 n} & \bar{z}_{n+1}^{m}
\end{array}\right)
\end{aligned}
$$

where we have introduced $z_{n+1}=z$. Since a particular enumeration of $z_{\ell}(1 \leq \ell \leq$ $n+1)$ is irrelevant, this reduces to

$$
\begin{aligned}
\left\langle q_{2 n}, z^{m}\right\rangle_{S} & \propto \int \cdots \int d^{2} Z_{1} \cdots d^{2} Z_{n} d^{2} Z_{n+1} \prod_{\ell=1}^{n+1}\left(z_{\ell}-\bar{z}_{\ell}\right) w^{2}\left(z_{\ell}, \bar{z}_{\ell}\right) \\
& \times \operatorname{det}\left(\begin{array}{ccccc}
1 & z_{1} & \cdots & z_{1}^{2 n} & z_{1}^{m} \\
1 & \bar{z}_{1} & \cdots & \bar{z}_{1}^{2 n} & \bar{z}_{1}^{m} \\
\vdots & \vdots & & \vdots & \vdots \\
1 & z_{n+1} & \cdots & z_{n+1}^{2 n} & z_{n+1}^{m} \\
1 & \bar{z}_{n+1} & \cdots & \bar{z}_{n+1}^{2 n} & \bar{z}_{n+1}^{m}
\end{array}\right)
\end{aligned}
$$

The latter integrand obviously vanishes for $0 \leq m \leq 2 n$ thus completing the proof of Eq. (18).

(ii) Consider

$$
\begin{aligned}
\left\langle q_{2 n+1}, z^{m}\right\rangle_{S} & \propto \int d^{2} Z(\bar{z}-z) w^{2}(z, \bar{z}) \int \cdots \int d^{2} Z_{1} \cdots d^{2} Z_{n} \\
& \times \prod_{k<\ell}\left|z_{k}-z_{\ell}\right|^{2}\left|z_{k}-\bar{z}_{\ell}\right|^{2} \prod_{\ell=1}^{n}\left|z_{\ell}-\bar{z}_{\ell}\right|^{2} w^{2}\left(z_{\ell}, \bar{z}_{\ell}\right) \\
& \times\left[\bar{z}^{m}\left(z+\sum_{k=1}^{n}\left(z_{k}+\bar{z}_{k}\right)\right) \prod_{\ell=1}^{n}\left(z-z_{\ell}\right)\left(z-\bar{z}_{\ell}\right)\right. \\
& \left.-z^{m}\left(\bar{z}+\sum_{k=1}^{n}\left(z_{k}+\bar{z}_{k}\right)\right) \prod_{\ell=1}^{n}\left(\bar{z}-z_{\ell}\right)\left(\bar{z}-\bar{z}_{\ell}\right)\right] .
\end{aligned}
$$

Invoking reasoning we have used in (i), this is further reduced to

$$
\left\langle q_{2 n+1}, z^{m}\right\rangle_{S} \propto \int \cdots \int d^{2} Z_{1} \cdots d^{2} Z_{n} d^{2} Z_{n+1} \prod_{\ell=1}^{n+1}\left(z_{\ell}-\bar{z}_{\ell}\right) w^{2}\left(z_{\ell}, \bar{z}_{\ell}\right)
$$


Eigenvalue correlations in non-Hermitean symplectic random matrices

$$
\begin{gathered}
\times \operatorname{det}\left(\begin{array}{ccccc}
1 & z_{1} & \cdots & z_{1}^{2 n} & z_{1}^{m} \\
1 & \bar{z}_{1} & \cdots & \bar{z}_{1}^{2 n} & \bar{z}_{1}^{m} \\
\vdots & \vdots & & \vdots & \vdots \\
1 & z_{n+1} & \cdots & z_{n+1}^{2 n} & z_{n+1}^{m} \\
1 & \bar{z}_{n+1} & \cdots & \bar{z}_{n+1}^{2 n} & \bar{z}_{n+1}^{m}
\end{array}\right) \sum_{\ell=1}^{n+1}\left(z_{\ell}+\bar{z}_{\ell}\right) \\
\left.-\operatorname{det}\left(\begin{array}{ccccc}
1 & z_{1} & \cdots & z_{1}^{2 n} & z_{1}^{m+1} \\
1 & \bar{z}_{1} & \cdots & \bar{z}_{1}^{2 n} & \bar{z}_{1}^{m+1} \\
\vdots & \vdots & & \vdots & \vdots \\
1 & z_{n+1} & \cdots & z_{n+1}^{2 n} & z_{n+1}^{m+1} \\
1 & \bar{z}_{n+1} & \cdots & \bar{z}_{n+1}^{2 n} & \bar{z}_{n+1}^{m+1}
\end{array}\right)\right]
\end{gathered}
$$

The latter trivially vanishes for $0 \leq m \leq 2 n-1$. This completes our proof of Eq. (19).

One may also verify that the normalisation $r_{n}$ in Eqs. (10) and (11) is related to $A_{n}$ [Eq. (20)] as $r_{n}=A_{n+1} / A_{n}$.

\subsection{Gaussian weight}

While the representations obtained above are fairly useful to study, e.g., asymptotic properties of general skew-orthogonal polynomials and address the issue of universality of eigenvalue correlations in non-Hermitean random matrix theory at $\beta=4$, there is no need to resort to them for a simple Gaussian weight

$$
w_{G}^{2}(z, \bar{z})=\exp \left[-\frac{N}{1-\tau^{2}}\left(z \bar{z}-\frac{\tau}{2}\left(z^{2}+\bar{z}^{2}\right)\right)\right]
$$

that we will be interested in in what follows.

In this case, skew-orthogonal Hermite polynomials are simple:

$$
\begin{aligned}
& q_{2 k+1}(z)=\left(\frac{\tau}{2 N}\right)^{k+1 / 2} H_{2 k+1}\left(z \sqrt{\frac{N}{2 \tau}}\right) \\
& q_{2 k}(z)=\left(\frac{2}{N}\right)^{k} k ! \sum_{\ell=0}^{k}\left(\frac{\tau}{2}\right)^{\ell} \frac{1}{(2 \ell) ! !} H_{2 \ell}\left(z \sqrt{\frac{N}{2 \tau}}\right) .
\end{aligned}
$$

Here $H_{k}(z)$ are 'conventional' Hermite polynomials

$$
H_{k}(z)=\frac{2^{k}}{\sqrt{\pi}} \int_{-\infty}^{+\infty} d t e^{-t^{2}}(z+i t)^{k}
$$

orthogonal in the complex plane $z$ with respect to the measure $w^{2}(z, \bar{z}) d^{2} Z$ (Di Francesco et al, 1994):

$$
\int d^{2} Z w_{G}^{2}(z, \bar{z}) H_{k}\left(z \sqrt{\frac{N}{2 \tau}}\right) H_{\ell}\left(\bar{z} \sqrt{\frac{N}{2 \tau}}\right)=\frac{\pi\left(1-\tau^{2}\right)^{1 / 2}}{N} \frac{2^{k} k !}{\tau^{k}} \delta_{k \ell} .
$$

Indeed, straightforward calculation in Eqs. (10) and (11) confirms that skeworthogonality is met with

$$
r_{k}=2 \pi(1-\tau)^{3 / 2}(1+\tau)^{1 / 2} \frac{(2 k+1) !}{N^{2 k+2}} .
$$

I This weight may be thought of as originating from the matrix model $H=H_{1}+i v H_{2}$, with each of $H_{\sigma}(\sigma=1,2)$ being drawn from statistically independent Gaussian symplectic ensembles of Hermitean random matrices $P\left[H_{\sigma}\right] \propto \exp \left\{-\left[N /\left(1+\tau^{2}\right)\right] \operatorname{Tr}\left(H_{\sigma}^{2}\right)\right\}$; the parameter $v^{2}=(1-\tau) /(1+\tau)$ [see, e.g., Fyodorov, Khoruzhenko and Sommers (1997)]. 
Yet another, integral representation for $q_{2 k}(z)$ holds which is more suitable for our purposes. To derive it we introduce the function

$$
F_{k}(z)=\sum_{\ell=0}^{k}\left(\frac{\tau}{2}\right)^{\ell} \frac{1}{(2 \ell) ! !} H_{2 \ell}(z)
$$

and notice that it satisfies the differential equation

$$
(1+\tau) \frac{\partial F_{k}}{\partial z}-2 \tau z F_{k}(z)=-\tau\left(\frac{\tau}{2}\right)^{k} \frac{1}{(2 k) ! !} H_{2 k+1}(z) .
$$

The latter is readily verified by making use of the identity $H_{\ell}^{\prime}(z)=2 \ell H_{\ell-1}(z)$. Integrating out, we infer

$$
F_{k}(z)=\exp \left(\frac{\tau z^{2}}{1+\tau}\right)\left[\sigma_{k}-\frac{\tau}{1+\tau}\left(\frac{\tau}{2}\right)^{k} \frac{1}{(2 k) ! !} \int_{0}^{z} d z^{\prime} \exp \left(-\frac{\tau{z^{\prime}}^{2}}{1+\tau}\right) H_{2 k+1}\left(z^{\prime}\right)\right]
$$

where

$$
\sigma_{k}=\sum_{\ell=0}^{k}\left(\frac{\tau}{2}\right)^{\ell} \frac{H_{2 \ell}(0)}{(2 \ell) ! !}, \quad H_{2 \ell}(0)=(-1)^{\ell} \frac{(2 \ell) !}{\ell !} .
$$

Summation over $\ell$ can be performed explicitly resulting in

$$
\sigma_{k}=\frac{1}{\sqrt{1+\tau}}\left[1-\frac{\tau^{k+1}}{2^{2 k+2} k !} H_{2 k+2}(0) \int_{0}^{1} \frac{d \xi \xi^{k}}{\sqrt{1+\tau \xi}}\right] .
$$

Taken together with Eqs. (23) and (25), this brings us to an exact integral representation for the even-order skew-orthogonal polynomials:

$$
\begin{aligned}
q_{2 k}(z) & =\left(\frac{2}{N}\right)^{k} \frac{k !}{\sqrt{1+\tau}} \exp \left(\frac{N z^{2}}{2(1+\tau)}\right) \\
& \times\left\{\left[1-\frac{\tau^{k+1}}{2^{2 k+2} k !} H_{2 k+2}(0) \int_{0}^{1} \frac{d \xi \xi^{k}}{\sqrt{1+\tau \xi}}\right]-\frac{\tau}{\sqrt{1+\tau}}\right. \\
& \left.\times\left(\frac{\tau}{2}\right)^{k} \frac{1}{(2 k) ! !} \sqrt{\frac{N}{2 \tau}} \int_{0}^{z} d w \exp \left(-\frac{N w^{2}}{2(1+\tau)}\right) H_{2 k+1}\left(w \sqrt{\frac{N}{2 \tau}}\right)\right\} .
\end{aligned}
$$

\section{Eigenvalue correlations in $\beta=4$ Gaussian ensembles}

In this section, we apply our findings to explicitly work out $n$-point correlation function for $\beta=4$ non-Hermitean random matrix ensemble associated with the Gaussian weight $w_{G}^{2}(z, \bar{z})[$ Eq. (21)]. By letting the parameter $\tau$ tend to zero, a strongly non-Hermitean Ginibre's ensemble is recovered. Scaling $\tau$ with matrix size $N$ as $\tau=1-\alpha^{2} / 2 N$ where $\alpha \sim O(1)$ one accesses a regime of weak non-Hermiticity (Fyodorov, Khoruzhenko and Sommers, 1997, 1997a) which is known to coincide with a zero-dimensional sector of a supersymmetry theory of disordered systems with a direction (Efetov, 1997).

For other papers addressing non-Hermitean Gaussian ensembles of symplectic symmetry by field-theoretic ( $\sigma$-model) techniques see, e.g., a supersymmetry treatment by Kolesnikov and Efetov (1999) and a replica approach by Nishigaki and Kamenev (2002). Unfotunately, both techniques run into obstacles when one attempts to study $n$-point correlation function whilst replica $\sigma$-models seem to reliably provide asymptotic expansions only (Verbaarschot and Zirnbauer (1985), Kanzieper (2001)). 


\subsection{Finite- $N$ solution}

In accordance with Eq. (6), the prekernel [Eq. (77)] is the only entity needed to evaluate $n$-point correlation function. Equations (13), (22), (23) and (24) furnish the desired solution

$$
\begin{aligned}
\kappa_{N}\left(z, z^{\prime}\right) & =\frac{1}{2 \pi}\left(\frac{1}{1+\tau}\right)^{1 / 2}\left(\frac{N}{1-\tau}\right)^{3 / 2}\left[\sum_{k=0}^{N-1}\left(\frac{\tau}{2}\right)^{k+1 / 2} \frac{1}{(2 k+1) ! !}\right. \\
& \left.\times H_{2 k+1}\left(z \sqrt{\frac{N}{2 \tau}}\right) \sum_{\ell=0}^{k}\left(\frac{\tau}{2}\right)^{\ell} \frac{1}{(2 \ell) ! !} H_{2 \ell}\left(z^{\prime} \sqrt{\frac{N}{2 \tau}}\right)-\left(z \leftrightarrow z^{\prime}\right)\right] .
\end{aligned}
$$

It holds for arbitrary finite $N$.

\subsection{Limit of infinite matrices: $N \rightarrow \infty$}

The large- $N$ limit is different for weakly and strongly non-Hermitean regimes.

5.2.1. Strong non-Hermiticity. As $\tau \rightarrow 0$, the prekernel simplifies to

$$
\kappa_{N}\left(z, z^{\prime}\right)=\frac{N^{3 / 2}}{2 \pi} \sum_{k=0}^{N-1}\left[\frac{(z \sqrt{N})^{2 k+1}}{(2 k+1) ! !} \sum_{\ell=0}^{k} \frac{\left(z^{\prime} \sqrt{N}\right)^{2 \ell}}{(2 \ell) ! !}-\left(z \leftrightarrow z^{\prime}\right)\right] .
$$

We are interested in a thermodynamic limit $N \rightarrow \infty$ with a blown-up energy resolution $z \mapsto z / \delta_{N}$ where $\delta_{N}=(N / 2 \pi)^{1 / 2}$. To this end we have to evaluate

$$
\begin{aligned}
\lim _{N \rightarrow \infty} \frac{1}{\delta_{N}^{3}} \kappa_{N}\left(\frac{z}{\delta_{N}}, \frac{z^{\prime}}{\delta_{N}}\right)=\sqrt{2 \pi} \\
\quad \times \sum_{k=0}^{\infty} \sum_{\ell=0}^{k}\left[\frac{(z \sqrt{2 \pi})^{2 k+1}}{(2 k+1) ! !} \frac{\left(z^{\prime} \sqrt{2 \pi}\right)^{2 \ell}}{(2 \ell) ! !}-\left(z \leftrightarrow z^{\prime}\right)\right] .
\end{aligned}
$$

An extra power of $\delta_{N}$ in the denominator of the l.h.s. is brought about by a prefactor $(\bar{z}-z)^{1 / 2}\left(\bar{z}^{\prime}-z^{\prime}\right)^{1 / 2}$ in Eq. (7).

Double summation in Eq. (29) can be performed explicitly. Denoting

$$
\sigma\left(z, z^{\prime}\right)=\sum_{k=0}^{\infty} \sum_{\ell=0}^{k}\left[\frac{z^{2 k+1}}{(2 k+1) ! !} \frac{\left(z^{\prime}\right)^{2 \ell}}{(2 \ell) ! !}-\left(z \leftrightarrow z^{\prime}\right)\right],
$$

we observe that

$$
\frac{\partial \sigma}{\partial z}=z \sigma+e^{z z^{\prime}}, \quad \frac{\partial \sigma}{\partial z^{\prime}}=z^{\prime} \sigma-e^{z z^{\prime}} .
$$

This suggests that we look for $\sigma\left(z, z^{\prime}\right)$ in the form

$$
\sigma\left(z, z^{\prime}\right)=e^{\frac{1}{2}\left(z^{2}+z^{\prime 2}\right)} \Lambda\left(z, z^{\prime}\right) .
$$

As soon as

$$
\frac{\partial \Lambda}{\partial z}=e^{-\frac{1}{2}\left(z-z^{\prime}\right)^{2}}, \quad \frac{\partial \Lambda}{\partial z^{\prime}}=-e^{-\frac{1}{2}\left(z-z^{\prime}\right)^{2}},
$$

we obtain

$$
\Lambda\left(z, z^{\prime}\right)=\int_{0}^{z-z^{\prime}} d t e^{-t^{2} / 2}=\sqrt{\frac{\pi}{2}} \operatorname{erf}\left(\frac{z-z^{\prime}}{\sqrt{2}}\right)
$$


This results in

$$
\lim _{N \rightarrow \infty} \frac{1}{\delta_{N}^{3}} \kappa_{N}\left(\frac{z}{\delta_{N}}, \frac{z^{\prime}}{\delta_{N}}\right)=\pi \exp \left[\pi\left(z^{2}+{z^{\prime}}^{2}\right)\right] \operatorname{erf}\left[\sqrt{\pi}\left(z-z^{\prime}\right)\right] .
$$

The latter is sufficient to evaluate all $n$-point correlation functions by means of Eqs. (6) and (7). For instance, the scaled density of states [Eqs. (5) and (14)] reads

$$
\begin{aligned}
\rho_{1}^{(4)}(z) & =(\bar{z}-z) \lim _{N \rightarrow \infty} \frac{1}{\delta_{N}^{3}} w^{2}\left(\frac{z}{\delta_{N}}, \frac{\bar{z}}{\delta_{N}}\right) \kappa_{N}\left(\frac{z}{\delta_{N}}, \frac{\bar{z}}{\delta_{N}}\right) \\
& =8 \pi Y^{2} \exp \left(-4 \pi Y^{2}\right) \int_{0}^{1} d \lambda \exp \left(4 \pi Y^{2} \lambda^{2}\right),
\end{aligned}
$$

$Y=\Im \mathrm{m} z$. A particular rescaling used in Eq. (29) has been chosen in such a way that the scaled level density $\rho_{1}^{(4)}(z)$ approaches unity at infinity, $|Y| \rightarrow \infty$.

5.2.2. Weak non-Hermiticity. The regime of weak non-Hermiticity is of a particular interest due to its close relation to Efetov's model of disordered systems with a direction. We reiterate that a degree of (weak) non-Hermiticity is governed by a parameter $\tau$ which scales with the matrix size $N$ as $\tau=1-\alpha^{2} / 2 N, \alpha \sim O(1)$.

The large- $N$ limit of the sum Eq. (13) [or Eq. (27)] is dominated by contributions of terms with $k$ such that $k / N \sim O(1)$. One therefore needs the asymptotics of skew orthogonal polynomials $q_{k}(z)$ at large indices $k$.

Asymptotics for odd-order skew-orthogonal polynomials $q_{2 k+1}$ are those of Hermite polynomials $H_{2 k+1}$ [Eq. 222] ]. Utilising the result from standard reference book by Szegö (1939)

$$
H_{2 k+1}\left(\frac{z}{2 \sqrt{k}}\right) \simeq \frac{2^{2 k+1}(-1)^{k} k !}{\sqrt{\pi k}} \sin z,
$$

we conclude that

$$
q_{2 k+1}(z) \simeq \frac{2^{2 k+1}(-1)^{k} k !}{\pi^{1 / 2}}\left(\frac{\tau}{2 N}\right)^{k+1 / 2} \sin \left(z \sqrt{\frac{2 k N}{\tau}}\right) .
$$

Here $k / N \sim O(1)$ and $z N \sim O(1)$.

Asymptotics for even-order skew-orthogonal polynomials $q_{2 k}$ can be read out from Eq. (26). Since

$$
\int_{0}^{1} \frac{d \xi \xi^{k}}{\sqrt{1+\tau \xi}} \simeq \frac{1}{k \sqrt{1+\tau}}, \quad k \gg 1
$$

we derive

$$
q_{2 k}(z) \simeq\left(\frac{2}{N}\right)^{k} \frac{k !}{\sqrt{1+\tau}}\left[1-\frac{\tau}{\sqrt{1+\tau}}\left(\frac{\tau}{2}\right)^{k+1} \frac{1}{(2 k+2) ! !} H_{2 k+2}\left(z \sqrt{\frac{N}{2 \tau}}\right)\right] .
$$

Applying further the asymptotic formula (Szegö, 1939)

we deduce

$$
H_{2 k}\left(\frac{z}{2 \sqrt{k}}\right) \simeq \frac{2^{2 k}(-1)^{k} k !}{\sqrt{\pi k}} \cos z
$$

$$
q_{2 k}(z) \simeq\left(\frac{2}{N}\right)^{k} \frac{k !}{\sqrt{1+\tau}}\left[1+\frac{(-1)^{k} \tau^{k+1}}{\sqrt{1+\tau} \sqrt{\pi k}} \cos \left(z \sqrt{\frac{2 k N}{\tau}}\right)\right]
$$


Equations (32) and (34) for skew-orthogonal Hermite polynomials at $k \gg 1$ make it now possible to evaluate the large- $N$ prekernel. Substituting the two equations into Eq. (13), and replacing the sum over $k$ by an integral we come up with

$$
\lim _{N \rightarrow \infty} \frac{1}{\delta_{N}^{3}} \kappa_{N}\left(\frac{z}{\delta_{N}}, \frac{z^{\prime}}{\delta_{N}}\right)=-\frac{\pi^{3 / 2}}{4 \alpha^{3}} \int_{0}^{1} \frac{d \lambda}{\lambda} e^{-\alpha^{2} \lambda^{2}} \sin \left[\pi\left(z-z^{\prime}\right) \lambda\right] .
$$

When taking the limit $N \rightarrow \infty$, the scale $\delta_{N}$ has been set to $\delta_{N}=N \sqrt{2} / \pi$.

In accordance with Eq. (6), knowledge of the scaled prekernel Eq. (35) is selfsufficient to have evaluated all $n$-point correlation functions. For instance, the density of states reads [Eqs. ([5) and (14)]

$$
\rho_{1}^{(4)}(z)=\frac{\pi^{3 / 2}}{2 \alpha^{3}} Y \exp \left(-\pi^{2} Y^{2} / \alpha^{2}\right) \int_{0}^{1} \frac{d \lambda}{\lambda} \exp \left(-\alpha^{2} \lambda^{2}\right) \sinh [2 \pi Y \lambda] .
$$

\section{Conclusions}

A problem of eigenvalue correlations in symplectic ensembles of non-Hermitean random matrices has exactly been solved by the method of orthogonal polynomials. In close analogy with $\beta=4$ Hermitean matrix ensembles, the $n$-point correlation function is given by a quaternion determinant [Eq. (6)] of an $n \times n$ matrix whose entries are quaternions with an image given by $2 \times 2$ matrices in the form of Eq. (7). To evaluate the latter it is convenient (but not obligatory) to introduce a set of polynomials which are skew-orthogonal in the complex plane [Eqs. (10) and (11)]. The skew-orthogonality set by Eq. (12) represents a natural basis in which calculational technology is most economic.

In Gaussian random matrix ensembles, the eigenvalue correlations are described by the prekernel Eq. (27) which further simplifies down to Eqs. (30) and (35) for strong and weak non-Hermiticity, respectively. These results apply not too close to the spectrum edges, which may also be studied within the current framework.

Remarkably, at $\beta=4$, all $n$-point spectral correlation functions exhibit a peculiar depletion of eigenvalues along the real axis $\Im \mathrm{m} z_{\ell}=0,1 \leq \ell \leq n$, where correlations vanish for both arbitrary matrix size $N$ and a probability measure $w^{2}(z, \bar{z})$. As for the remaining nontrivial functional dependence, we expect it to be universal as well once a thermodynamic limit is taken. Equations (18) and (19) will obviously serve as a proper starting point to address the universality issue in either the spectrum bulk or near the complex edges of the eigenvalue support.

Acknowledgments. I wish to thank P. J. Forrester and M. B. Hastings for a number of useful comments and references.

\section{References}

Bruijn de N G 1955 On some multiple integrals involving determinants, J. Indian Math. Soc. 19133

Dyson F J 1972 Quaternion determinants, Helv. Phys. Acta 45289

Edelman A 1997 The probability that a random real Gaussian matrix has $k$ real eigenvalues, related distributions, and the circular law, J. Mult. Analysis 60203

Efetov K B 1997 Quantum disordered systems with a direction, Phys. Rev. B 569630

Eynard B 2001 Asymptotics of skew orthogonal polynomials, J. Phys. A: Math. Gen. 347591

Francesco Di F, Gaudin M, Itzykson C and Lesage F 1994 Laughlin wave-functions, Coulomb gases and expansions of the discriminant, Int. J. Mod. Phys. A 94257

Fyodorov Y V, Khoruzhenko B A and Sommers H-J 1997 Almost Hermitean random matrices: Eigenvalue density in the complex plane, Phys. Lett. A 22646 
Fyodorov Y V, Khoruzhenko B A and Sommers H-J 1997a Almost Hermitean random matrices: Crossover from Wigner-Dyson to Ginibre eigenvalue statistics, Phys. Rev. Lett. 79557

Fyodorov Y V, Khoruzhenko B A and Sommers H-J 1998 Universality in the random matrix spectra in the regime of weak non-Hermiticity, Ann. Inst. Henri Poincare (Physique Theorique) 68449

Ginibre J 1965 Statistical ensembles of complex, quaternion, and real matrices, J. Math. Phys. 6440

Halasz M A, Osborn J C and Verbaarschot J J M 1997 Random matrix triality at nonzero chemical potential, Phys. Rev. D $\mathbf{5 6} 7059$

Hastings M B 2000 Fermionic mapping for eigenvalue correlation functions of weakly non-Hermitian symplectic ensemble, Nucl. Phys. B 572535

Kanzieper E and Freilikher V 1999 Spectra of large random matrices: A method of study, in: Diffuse Waves in Complex Media, ed. by J.-P. Fouque, NATO ASI, Series C (Math. and Phys. Sciences) 531165 (Kluwer, Dordrecht)

Kanzieper E 2001 Random matrix theory and the replica method, Nucl. Phys. B 596548

Kolesnikov A V and Efetov K B 1999 Distribution of complex eigenvalues for symplectic ensembles of non-Hermitian matrices, Waves in Random Media 971

Lehmann N and Sommers H-J 1991 Eigenvalue statistics of random real matrices, Phys. Rev. Lett. 67941

Mahoux G and Mehta M L 1991 A method of integration over matrix variables: IV, J. Phys. I France 11093

Mehta M L 1967 Random Matrices (New York: Academic Press)

Mehta M L and Gaudin M 1960 On the density of eigenvalues of a random matrix, Nucl. Phys. B 18420

Nishigaki S M and Kamenev A 2002 Replica treatment of non-Hermitian disordered Hamiltonians, J. Phys. A: Math. Gen. 354571

Oas G 1997 Universal cubic eigenvalue repulsion for random normal matrices, Phys. Rev. E 55205

Szegö G 1939 Orthogonal Polynomials (Providence: AMS)

Tracy C A and Widom H 1998 Correlation functions, cluster functions and spacing distributions for random matrices, J. Stat. Phys. 92809

Verbaarchot J J M and Zirnbauer M R 1985 Critique of the replica trick, J. Phys. A: Math. Gen. 18 1093 